ENCYCLOPÉDIE Encyclopédie berbère

BERBERE

$7 \mid 1989$

7 | Asarakae - Aurès

\title{
Attici Ag Amellal
}

(Atīsi)

M. Bourgeot et M. Gast

\section{OpenEdition}

Journals

Édition électronique

URL : http://journals.openedition.org/encyclopedieberbere/1217

DOI : 10.4000/encyclopedieberbere. 1217

ISSN : 2262-7197

\section{Éditeur}

Peeters Publishers

\section{Édition imprimée}

Date de publication : 1 janvier 1989

Pagination : 1043-1046

ISBN : 2-85744-443-5

ISSN : $1015-7344$

\section{Référence électronique}

M. Bourgeot et M. Gast, « Attici Ag Amellal », Encyclopédie berbère [En ligne], 7 | 1989, document A315, mis en ligne le 01 décembre 2012, consulté le 13 octobre 2020. URL : http://journals.openedition.org/ encyclopedieberbere/1217; DOI : https://doi.org/10.4000/encyclopedieberbere.1217

Ce document a été généré automatiquement le 13 octobre 2020

(c) Tous droits réservés 


\title{
Attici Ag Amellal
}

(Atīsi)

\author{
M. Bourgeot et M. Gast
}

\section{(A. Bourgeot)}

1 Attici Ag Amellal, huitième aménokal dans la lignée des chefs traditionnels des Kel Ahaggar, régna de 1900 à 1904 dans des conditions historiques houleuses qui correspondaient à la pénétration française en Ahaggar par les armes, et dans des conditions politiques internes particulièrement tendues. En effet, selon les règles qui président à la transmission du pouvoir politique, aurait dû être désigné comme successeur légal de Aytayel* Ag Mohamed Biska, septième aménokal, le fils aîné de sa sœur aînée Khetova : Mohamed Ag Urzig.

2 Or, cette succession s'engagea dans un contexte particulièrement sombre. En effet, en 1899, la mission Flamand-Pein après la prise d'In Salah entreprit la conquête du Tidikelt, oasis et territoires qui étaient dans la mouvance et sous la domination des Kel Ahaggar. Ceux-ci ressentaient donc directement la pression française qui les amputait déjà de leur zone d'influence en les privant de leurs marchés septentrionaux. Par ailleurs, ils envisageaient clairement la venue imminente des troupes françaises lesquelles étaient perçues comme un danger majeur capable de remettre en cause leur hégémonie militaire et politique. Ce danger immédiat engendra un clivage politique à l'intérieur de la société des imuhay qui se divisa en deux tendances opposées: l'une, minoritaire et partisante de la collaboration avec les Français se regroupa autour de Mohamed Ag Urzig (c'est au sein de cette tendance que se dessinait déjà « le parti de la France » groupé autour de Moussa Ag Amastan) ; l'autre, majoritaire, se constitue en parti hostile à la colonisation autour de Attici Ag Amellal fils aîné de la sœur cadette d'Aytayel, (Khaouila oult Mohamed Biska).

3 L'Ameni, assemblée des notables composée d'Ihaggaren (suzerains) et d'imyad (tributaires) chargée de contrôler le bon fonctionnement de la transmission du pouvoir se prononça très nettement en faveur d'Attici Ag Amellal représentant le parti de la résistance à la pénétration française. Il est intéressant de souligner que la totalité des 
imyad siégeant à l'ameni se prononça pour Attici, tandis que seule une minorité des Ihaggaren abonda dans ce sens. Ce dernier point met en évidence les contradictions qui existaient au sein de la classe dominante.

Il apparaît clairement que la succession à l'aménokalat se pose en termes politiques en relation avec la position à adopter face à l'envahisseur. C'est donc dans ce contexte de conquête (il en allait de l'hégémonie touarègue au Sahara central) qu'intervient le poids de l'Islam et la fonction des ineslimen (les religieux). En effet, ce fut 'Abidine, marabout particulièrement influent qui, désirant éviter un clivage trop grave au sein des Ihaggaren, neutralisa provisoirement le conflit en créant un pouvoir bicéphale assuré d'un côté par Mohamed Ag Urzig, et de l'autre, par Attici Ag Amellal. Cette création fut immédiatement entérinée par un geste symbolique réalisé par 'Abidine qui déchira son voile de tête en deux parties et plaça chaque moitié sur les têtes d'Attici et de Mohamed. Il est bien évident qu'une telle situation était en contradiction avec les mécanismes de centralisation et de renforcement du pouvoir politique. Cette contradiction favorisa très rapidement un climat d'anarchie qui se manifesta à propos du prélèvement du tribut (la tiousé) sur les imүad. En effet, les tenants du pouvoir prélevaient chacun de leur côté la tiousé sur les imyad afin de confirmer leur fonction et leur pouvoir sur les tributaires.

5 Les imghad, sur-exploités et lassés de cet état d'anarchie dont ils furent les principales victimes, tournèrent casaque et orientèrent leurs démarches vers le " parti de la paix " en nouant de nouveaux contacts avec Moussa Ag Amastan*. C'est à ce moment précis que réapparaît et rebondit le problème de la succession à travers les alliances et les inimitiés antérieures.

6 Attici et son frère cadet Anaba, appartenaient aux Teğéhê Mellet par leur père et aux Kel yela par leur mère. Au moment de l'extermination de la colonne Flat-ters, ils étaient affiliés aux Teğéhé Mellet puis s'intégrèrent aux Kel yela à la mort de leur père. Ils se montrèrent farouchement déterminés contre l'intervention de toute puissance étrangère, notamment contre celle de la France.

7 Attici participa directement à la destruction de la colonne conduite par le colonel Flatters. Après le combat de Tit, en 1902, qui vit le début du déclin de la puissance guerrière touarègue et l'apparition de Moussa Ag Amastan sur la scène politique, Attici et ses partisans poursuivirent leur politique hostile à la pénétration française. C'est ainsi qu'en juin 1904 le "parti du refus » composé d'Attici et de ses partisans réussit à faire échouer l'entrevue qui devait avoir lieu dans l'Adrar-n-Ifoyas (dans l'actuel Mali) entre Moussa Ag Amastan et le commandant Laperrine. Par la suite, il ne perdit pas une occasion d'affirmer son autorité et son opposition. Malgré son éviction du pouvoir, il continua à prélever l'impôt sur les dépendants Iseqqamaren et intervint directement dans les affaires judiciaires chez les nomades de la Tefedest, notamment à propos de meurtres dont le mobile demeure obscur. Il fallut plusieurs années pour éliminer son influence politique.

\section{Discussion (M. Gast)}

- Le parti de Mohamed ag Urzig n'était pas le "parti de la France " groupé autour de Moussa; personne ne parlait en 1900 de Moussa ag Amastan comme d'un éventuel amenoûkal, bien que Benhazera ait écrit qu'Aïtayel l'aurait désigné comme successeur, 
on peut attribuer cette rumeur diffusée après coup en fonction de la renommée de Moussa. Il était bien trop jeune en 1900 et devait avoir environ 27 ans. Sa forte personnalité morale et guerrière n'a été un recours qu'après la bataille de Tit en 1902, surtout devant l'état de désarroi politique et économique engendré par les deux amenoûkal concurrents Attici et Mohamed (voir Benhazera, 1908, p. 126-127).

- C'est Mohamed ag Urzig, pour nuire à Attici dont l'autorité lui était supérieure, qui envoya Baba ag Tamoklast en rezzou contre le campement de M'hammed ben Messis près d'In Salah. Cette entreprise était une véritable provocation, grossière et téméraire, destinée à déclencher un " gros coup » contre les Kel Ahaggar. Le commandant Cauvet n'a fait que céder aux demandes pressantes des Mrabtines d'In Salah en les laissant organiser un contre-rezzou qui déclencha la bataille de Tit en 1902.

- Attici et Anaba (ag Chikkat dit Amellal) n'étaient pas «affiliés » aux Teğehe Mellet puis aux Kel yela. Ils jouaient, selon les lieux et les époques, de l'une ou l'autre de leur filiation. Hostiles à leur oncle maternel l'amenoûkal Aytayel, c'est eux qui déclenchèrent le massacre de la mission Flatters en 1880 pour créer des ennuis à Aytayel et provoquer sa succession dont ils étaient parmi les premiers bénéficiaires potentiels. Le commandement militaire français de l'époque (à Ouargla et Alger) ne comprit pas les dénégations des Kel yela et d'Aytayel dans la participation à cette affaire que tous les envoyés de l'Ahaggar déclaraient être le fait des Ouled Messaoud (voir Archives Outre Mer, Aix-en-Provence). Or, il faut savoir que ces Ouled Messaoud, en langue arabe d'In Salah, ce sont ceux que les Touareg appellent les Teğehe Mellet dont se réclamaient Attici et Anaba de par leur père. Les Teğehe Mellet avaient en effet beaucoup de liens de parenté avec des Iforas d'In Salah et d'ailleurs.

11 En outre, si Aytayel n'a jamais sévi contre Attici et Anaba dont il connaissait tous les méfaits, c'est parce qu'ils étaient les fils de sa sœur et que les règles de relations parentales d'oncle maternel à neveu lui interdisaient toute cœrcition. Aytayel bien qu'amenoûkal, restait donc prisonnier des agissements de ses neveux qui n'avaient de cesse de l'éliminer, mais qui se nourrissaient constamment chez lui, comme le veut la règle traditionnelle.

Dès qu'Aytayel disparaît, Attici, l'aîné, rappelle qu'il est Kel yela par sa mère et héritier du commandement de son oncle maternel. On peut dire que dans son cas la double filiation â été une réalité historique, politique et sociale et qu'il s'en est servi selon les circonstances et l'opportunité de ses intérêts.

- Moussa ag Amastan a accédé très jeune (vers 32 ans) au commandement suprême non pas en raison de la force machiavélique des agents militaires français, mais surtout grâce à la conjugaison de trois ensembles de faits.

1. Son oncle Khyar ag Hegyer rêvait de devenir amenoûkal, s'y était préparé toute sa vie, mais n'avait pas vécu des temps qui lui furent favorables. Il investit une partie de son savoir et de son habileté politique chez Moussa qui aurait pu être son successeur et qu'il aimait comme un fils.

2. Moussa, enfant pauvre, mais intelligent et courageux, vécu une partie de sa vie dans l'Adrar au contact intime des Iforas et des Atouaj, tribus maraboutiques qu'il protégea souvent quand il devint un guerrier renommé. Par la suite Moussa devint très religieux et fut toujours conseillé et soutenu par tous les religieux (en particulier Bey el Bekkaye des Kounta, chef de la Zaouia de Teleya) ; ce sont eux surtout qui l'ont élevé au pouvoir, eux qui l'ont poussé à aller à In Salah voir les Français, eux qui ont préparé son retour 
dans l'Ahaggar pour qu'il soit proclamé amenoûkal. Il est vrai qu'une partie de ces marabouts était aussi manipulée par les militaires français à In Salah. Mais d'un côté comme de l'autre ces marabouts défendaient leurs réseaux d'autorité et leur survie.

3. Si Mohamed ag Urzig et Attici avaient été plus habiles pour s'entendre, il n'y aurait pas eu la bataille de Tit et s'il n'y avait pas eu la menace d'éclatement de la confédération des Touaregs de l'Ahaggar, Moussa ag Amastan n'aurait jamais pu être amenoûkal en de pareilles circonstances. Evénements engendrés en partie par la colonisation certes, mais aussi par d'autres circonstances internes à l'organisation touarègue.

\section{La fin dAttici}

14 L'histoire orale n'a guère conservé la mémoire de ce que devint Attici après l'entrée en scène de Moussa ag Amastan. On sait qu'il était riche (c'est-à-dire qu'il avait suffisamment de troupeaux). Il rejoint Kaocen en 1916 lors de la dissidence senoussiste. Après 1917 et la défaite de Kaocen tous ses partisans les plus notoires se dispersèrent et entrèrent dans l'anonymat en émigrant en partie en Libye. Il est probable qu'Attici demeura au Tamesna ou à la périphérie de l'Ahaggar sans jamais plus refaire parler de lui.

\section{BIBLIOGRAPHIE}

BENHAZERA M., Six mois chez les Touareg du Ahaggar, Alger, A. Jourdan, 1908, p. 223.

BISSUEL H., Les Touaregs de l'ouest, Alger, A. Jourdan, 1888, p. 210, cartes, index.

BOURGEOT A., « Contribution à l'étude de la parenté touarègue », Aix-en-Provence, Rev. de l'Occ.

musulman et de la Méditerranée, n²2, 1976, 9-31, p. 23 à 29.

BUTAYE P., « Le droit au commandement chez les Kel Ahaggar », Alger, Bull, de Liaison Sahar, $\mathrm{n}^{\circ} 28$,

1957, p. 250-256.

CAUVET G., Le raid du lieutenant Cottenest au Hoggar, combat de Tit, 7 mai 1902, Marseille, R. et J.

Brunon 1945, p. 146, cartes, photo, annexes, p. 79.

DELON, L'amenokalat dans les confédérations touareg, Mém. du CHEAM, 1951, nº 1863.

FOUCAUld Ch. de., Dictionnaire Touareg-Français, Impr. Nationale de France, 1951, t. II, p. 916.

GAST M., « The History of sovereignty among the Kel Ahaggar » in the Nomadic alternative, W.

Weissleder édit. Mouton, the Hague, Paris, 1978, p. 201-213.

RÉGNIER, «A propos du commandement chez les Kel Ahaggar », Alger, Bull, de Liaison Sahar, $\mathrm{n}^{\circ} 41$, 1961, p. 55-65. 
INDEX

Mots-clés : Biographie, Histoire 